\title{
Eliciting and modelling expertise for serious games in project management
}

\author{
William Seager, Martin Ruskov, M. Angela Sasse \\ Department of Computer Science, University College London, London, UK \\ Manuel Oliveira \\ Sintef, Trondheim, Norway
}

\section{Abstract}

Without achieving a clear understanding of the learning domain, it is difficult to develop a successful serious game that enables users to achieve the desired learning outcomes. Thus, the first step in serious game design is to establish an understandding of the particular learning domain, usually through consultation with domain experts. Whilst game design is inherently a creative process, we believe the capturing of the knowledge domain can be systematised and we present a structured approach to knowledge elicitation and representation as a basis for serious game design. We have adapted and extended the applied cognitive task analysis (ACTA) method and have combined it with additional knowledge representation frameworks. We explain how the outputs of this approach can inform the game mechanic and the development of non-player characters, and apply it to the design of a serious game aimed at reducing time-to-competence in soft project management skills for professionals working in corporate environments. A total of 26 domain experts from five different countries were involved in a two-stage interview process. The interviews yielded more than 300 task elements, and information about the cognition underlying the more challenging tasks. This data was incorporated into several representation frameworks and used to indicate features to be implemented in the game and the game mechanics of the supported features.

\section{Introduction}

(Seager et al., 2010)

There is a growing interest in games for purposes beyond entertainment. Serious games are designed to facilitate learning whilst entertaining and engaging users. Examples have been developed in a wide range of fields including management science, economics, intercultural communication, psychology, military strategy, sociology, political science, and interpersonal skill development (Raybourn and Bos, 2005). The hypothesised benefits of digital game-based learning include increased motivation of learners, and improved transfer of learning to the context in which the learning is applied. According to one industry estimate, the market for serious games is likely to grow to approximately 700 million USD by 2010 as gaming emerges as a critical component in many corporate learning solutions (Filho and Latham, 2006). On the other hand, Microsoft announced on Business Week (Jana, 2007) that their estimates of the potential serious games US market approaches 9 billion USD, but it remains a challenge to reconcile the different estimates

Please, cite as: Seager, W., Ruskov, M., Fradinho, M., Sasse, A., 2010. Eliciting and Modelling Expertise for Serious Game Design. Entertainment Computing 2, 75-80.

doi:10.1016/j.entcom.2011.01.002 
with no concrete market statistics for the serious games market. However, if one considers the games industry market as a whole, the forecast is promising, as evidenced in the annual report of Entertainment Software Association (ESA) stating that the total sales in the US market alone for 2009 totalled 10.2 billion USD despite the current economic recession (ESA, 2010). Also, there is growing recognition of game based learning as a useful tool to support learning within schools and universities (Eck, 2006).

Unlike entertainment games, serious game development relies on a close collaboration between game designers and developers and domain experts, as game designers and developers are usually not familiar with the pedagogical content, whilst domain experts rarely have the skills to design and build games (Eck, 2006). Anecdotal evidence suggests that gathering and using information via traditional mechanisms such as focus groups and interviews for serious game design can be problematic. For example, in the case of the Virtual Leader serious game, plans for close collaboration with domain experts were eventually dropped when it became clear that the insights yielded would be difficult to integrate into the game. In the end, the game design relied on a crafted approach confined to the ideas of the developers (Aldrich, 2009).

We believe that game design will always to some extent resemble a craft-like process that involves a high degree of creativity. However, we think that the front-end part of the design process can be made more systematic and thus more reliable and predictable (Vidani and Chittaro, 2009). In particular, there is potential for systematic methodologies that can reliably distil pedagogical content from domain experts and represent it in a way that can be easily interpreted by game designers.

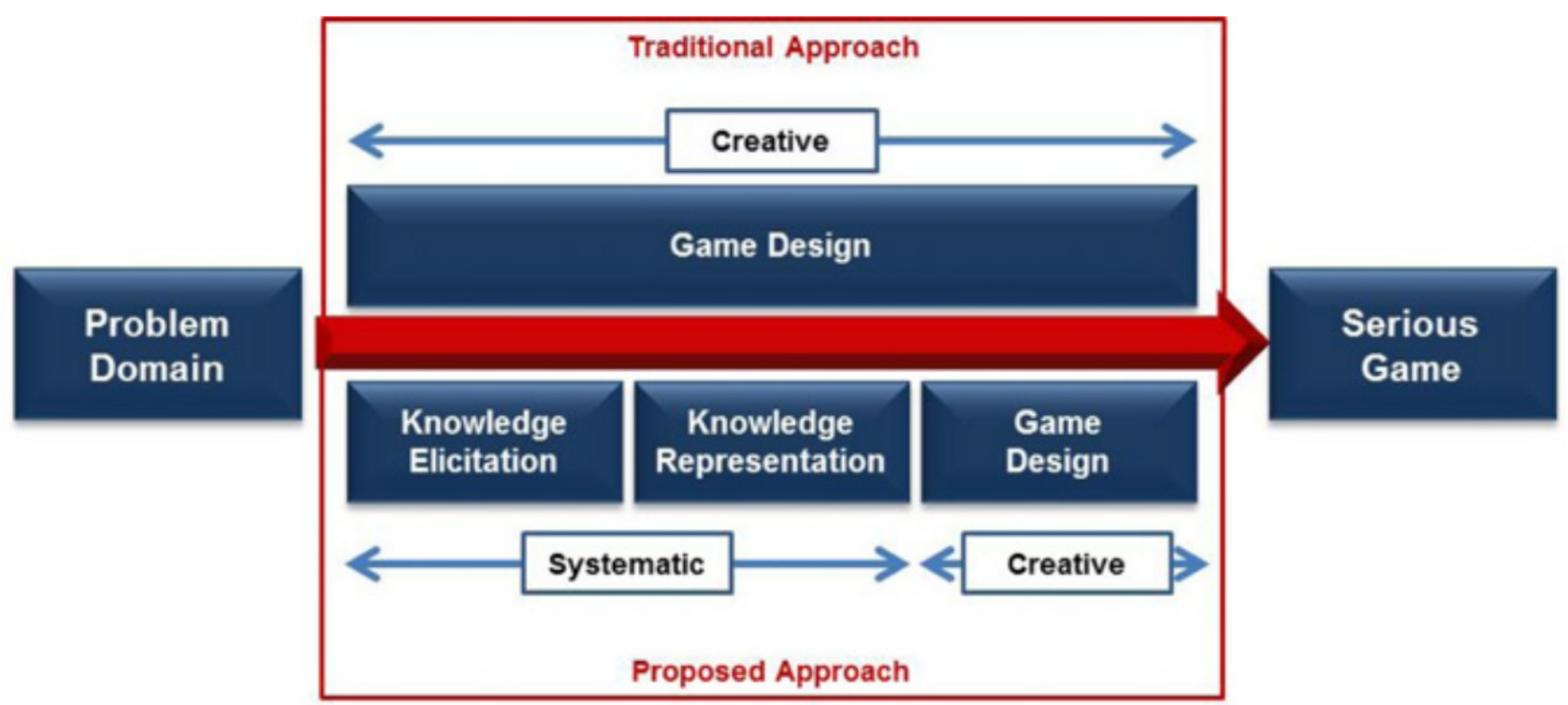

Figure 1: Traditional and proposed game design approaches.

In our approach, game design is segmented into three stages: knowledge elicitation, knowledge representation and game design (see Figure 1). Cognitive Task Analysis (CTA) is used to elicit and represent knowledge and information about thought processes in a systematic way (Chipman et al., 2000). We adopt a specific method called Applied Cognitive Task Analysis (ACTA) (Militello and Hutton, 1998). We adapt and extend ACTA by combining it with additional knowledge 
representation frameworks. Our approach allows us to:

1. Generate a scoped task model of the domain.

2. Identify task elements that novice learners often find particularly challenging.

3. Generate information about the knowledge, thought process and goal structures that underlie observable task performance in the domain.

\section{Related Research}

At present, the literature on digital game-based learning provides little guidance on how to design successful serious games. In particular, there are few systematic methodologies to guide and organise game design to ensure that production is more predictable, development time shorter, and to enhance the educational or instructional effect of games. Although we recognise that game design is inherently a creative process, we believe the capturing of the knowledge domain can be systematised to increase the likelihood of building a successful game.

One systematic approach to game design based on task modelling has been proposed (Vidani and Chittaro, 2009). This approach uses the ConcurTaskTrees (CCT) technique (Paterno et al., 1997) to analyse and structure pedagogical content about the procedures within a particular task domain. The method is applied to the design of a serious game for training nurses in emergency medical procedures. Whilst promising, this approach incurs the limitation that it only elicits information about procedural task elements. It does not elicit information about the knowledge, thought processes and goal structures that underlie task performance.

"Cognitive task analysis (CTA)" describes a set of methods that yield information about the knowledge, thought processes and goal structures that underlie observable task performance (Chipman et al., 2000). The three main components of CTA are knowledge elicitation, data analysis and knowledge representation. There are numerous knowledge elicitation methods (for a review, see (Crandall et al., 2006). Most CTA methods also provide methods for analysing and representing the data obtained through knowledge elicitation (Crandall et al., 2006).

Applications of CTA include system design, training design, human-computer interface design, accident investigation and the development of tests to assess competence (Baber et al., 2005; Chipman et al., 2000). CTA methods have been applied within a wide range of domains including aviation, nuclear power plant operation, consumer behaviour, air traffic control and military operations, and consumer research (Baber et al., 2005). A large number of distinct CTA techniques have been developed (Baskin and John, 1998; Zachary et al., 1998). Together, these techniques incorporate a considerable variety in approach, emphasis and resource requirements (Militello and Hutton, 1998).

Knowledge elicitation and representation techniques can also support the design of Non-Player Characters (NPCs) (Norling, 2009; Norling and Sonenberg, 2004). NPCs are a common feature of entertainment and serious games. One approach to developing agent-based NPCs draws on the BDI (Belief-Desire-Intention) framework (Bratman, 1987). The BDI framework has been used to create human-like characters in a range of computer-based simulations and games (e.g. (Lui et al., 2002). 
According to Norling (Norling, 2003), the BDI paradigm is well suited to the creation of synthetic characters, particularly those with human characteristics.

\section{Approach}

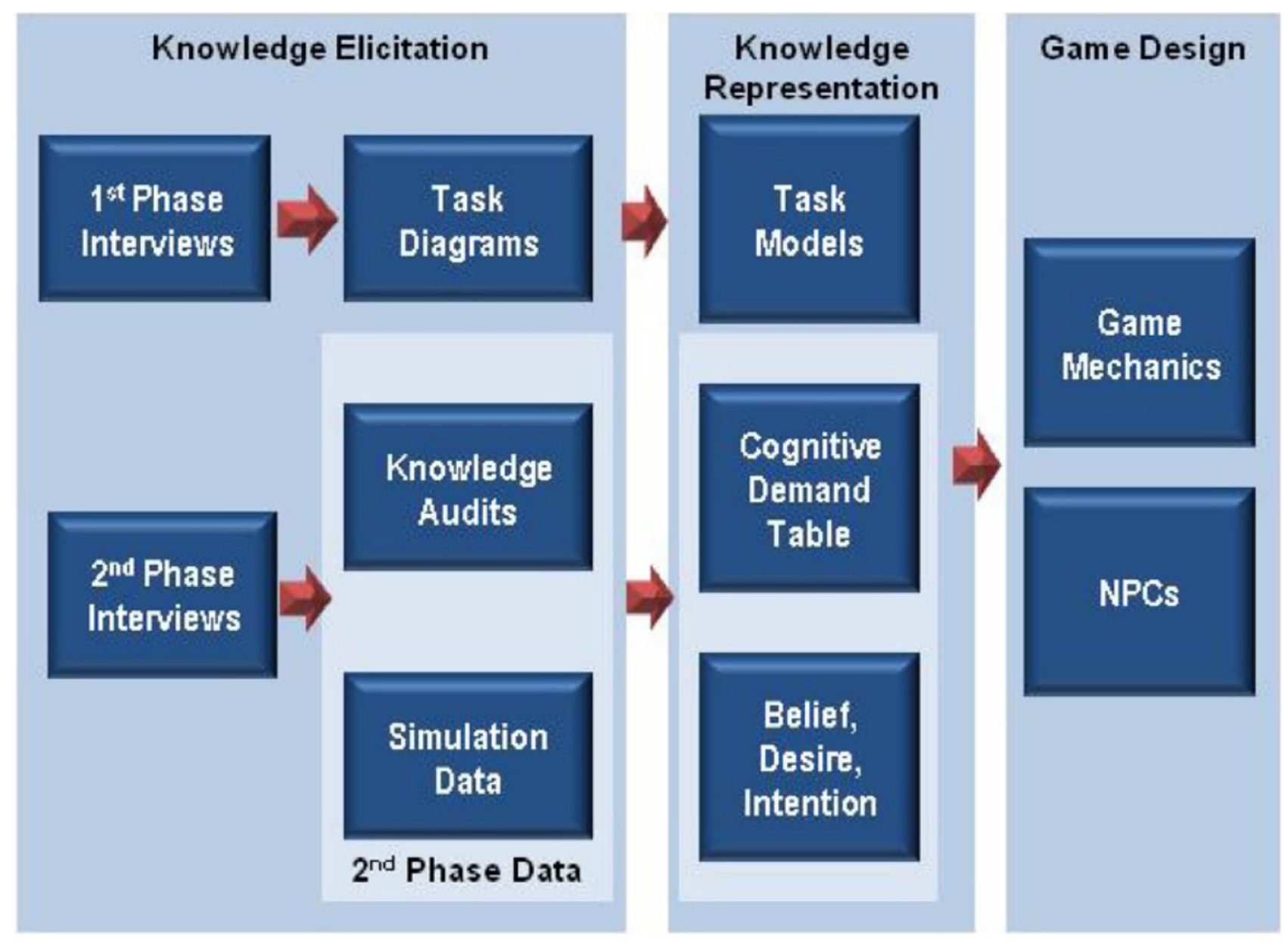

Figure 2: Our proposed approach.

The block diagram in Figure 2 below presents an overview of our proposed three-stage game design process. The stages in the process are knowledge elicitation, knowledge representation and game design. Our approach to knowledge elicitation is founded on a CTA method called Applied Cognitive Task Analysis (ACTA) (Militello and Hutton, 1998). ACTA provides three interview protocols: the task diagram interview, the knowledge audit, and the simulation interview. The task diagram interview elicits information about the task structure within a particular task domain (e.g. the main tasks and sub-tasks), and helps identify which of these task components are typically experienced as challenging or difficult. The knowledge audit and simulation interviews generally focus on the more difficult/challenging components and elicit more detailed information about the underlying knowledge, thought processes and goal structures. The main output of the ACTA method is the Cognitive Demands Table. This framework includes information about why each element is often found to be difficult, identifies common pitfalls/errors incurred by novices, and identifies cues and strategies that experts use to overcome the difficulties. In our approach, the Cognitive Demands Table is combined with a Task Model framework and the Belief, Desire Intention (BDI) framework (Bratman, 1987; Norling and Sonenberg, 2004). Figure 3 below shows in the abstract the overall 
knowledge representation framework that results from our approach.

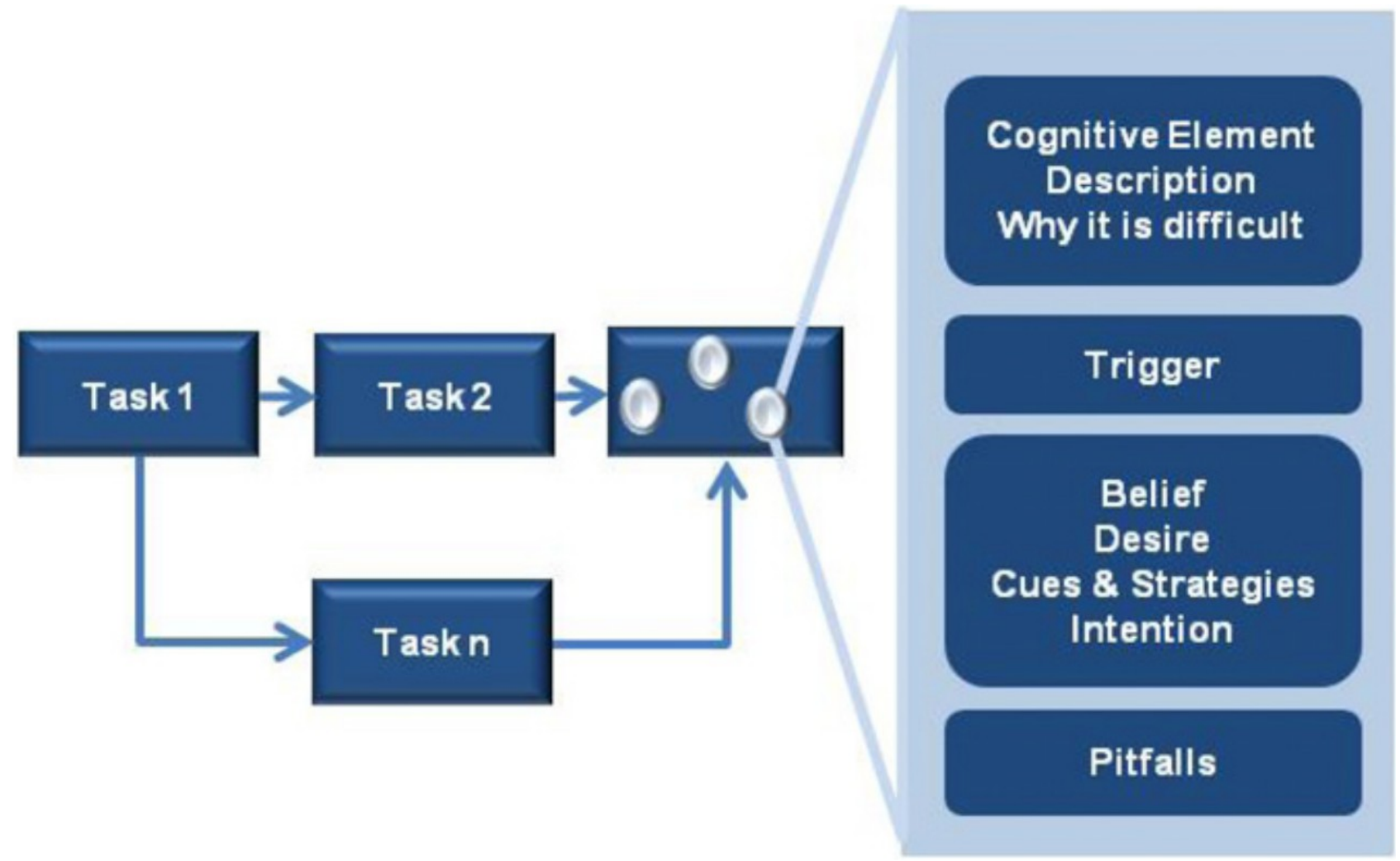

Figure 3: Overview of the model based on three knowledge representation frameworks.

The task structure on the left-hand side of the figure shows the output of the task diagram interviews: this includes the main sub-tasks within a particular task area, the typical procedural relationship between them, and the parts of each task that are considered to be the most cognitively challenging. The difficult cognitive elements are explored further in the knowledge audit and simulation interviews leading to the types of information shown in the right-hand side of the figure. The reason for using two representational frameworks for the data resulting from these interviews is that they capture different perspectives: the cognitive demands table is useful for determining game mechanics and flow, whilst the BDI technique provides the baseline for the development of pedagogical agents.

In the following sections, we illustrate our approach by applying it to the design of a serious game aimed at reducing time-to-competence in soft project management skills for professionals working in corporate environments. The resulting output is scoped to what is cognitively challenging, and contributes to the game design by helping to shape the game mechanics and NPC design.

\section{Knowledge elicitation}

The three ACTA interview techniques were applied in two phases of interviews. In the first phase, 16 experienced project managers were interviewed using the Task Diagram Protocol. Based on the results of this stage, 10 further in-depth interviews were carried out using the Knowledge Audit and Simulation Interviews. 


\section{Task diagram interviews}

The participants in the task diagram interviews were based in four different countries (4 in Norway, 3 in the UK, 6 in Bulgaria and 3 in Brazil). Fifteen were male and one was female. They were aged from 30 to 68 years. On average, they had 17 years project management experience (all had at least 5 years experience). Their project management experience was obtained from a variety of fields including civil engineering, software engineering, academic research, electronic engineering, and pharmaceutical research. The interviews were conducted by four interviewers. At the beginning of each interview, participants were briefed about the goals of the research. Following a short questionnaire, they were presented with a high-level task model of project management and a supporting glossary of terms. The model was developed by project management researchers based at the SINTEF Group in Norway and depicted twenty three task areas within project management and the typical dependencies between these task areas. Participants were asked to choose up to five tasks areas they considered to be important to the success of projects, and particularly challenging in terms of the cognitive skills required. Cognitive skills were defined as judgments, assessments, problem solving or thinking skills.

Each chosen task area was then addressed using the task diagram interview protocol. Using a whiteboard or PowerPoint slide, participants were asked to sub-divide each task area into 3-6 subtasks. They were then asked to specify which of the sub-tasks were particularly challenging in terms of the cognitive skills required. Each challenging sub-task was further sub divided into smaller task elements. The interviews took from 30 to 90 min to carry out, with most lasting approximately 60 min. The output of each interview was a set of task diagrams. Figure 4 presents a partial task diagram from one interview. In this diagram, the participant has chosen 'defining project goals' as one task area to address in the interview. He identifies four sub-tasks in this task area (Figure 4a) and indicates that one of these sub-tasks - stakeholder analysis - is cognitively challenging. He then sub-divides stakeholder analysis into smaller task elements, and identifies which of these elements are cognitively challenging (Figure $\mathbf{4 b}$ ).

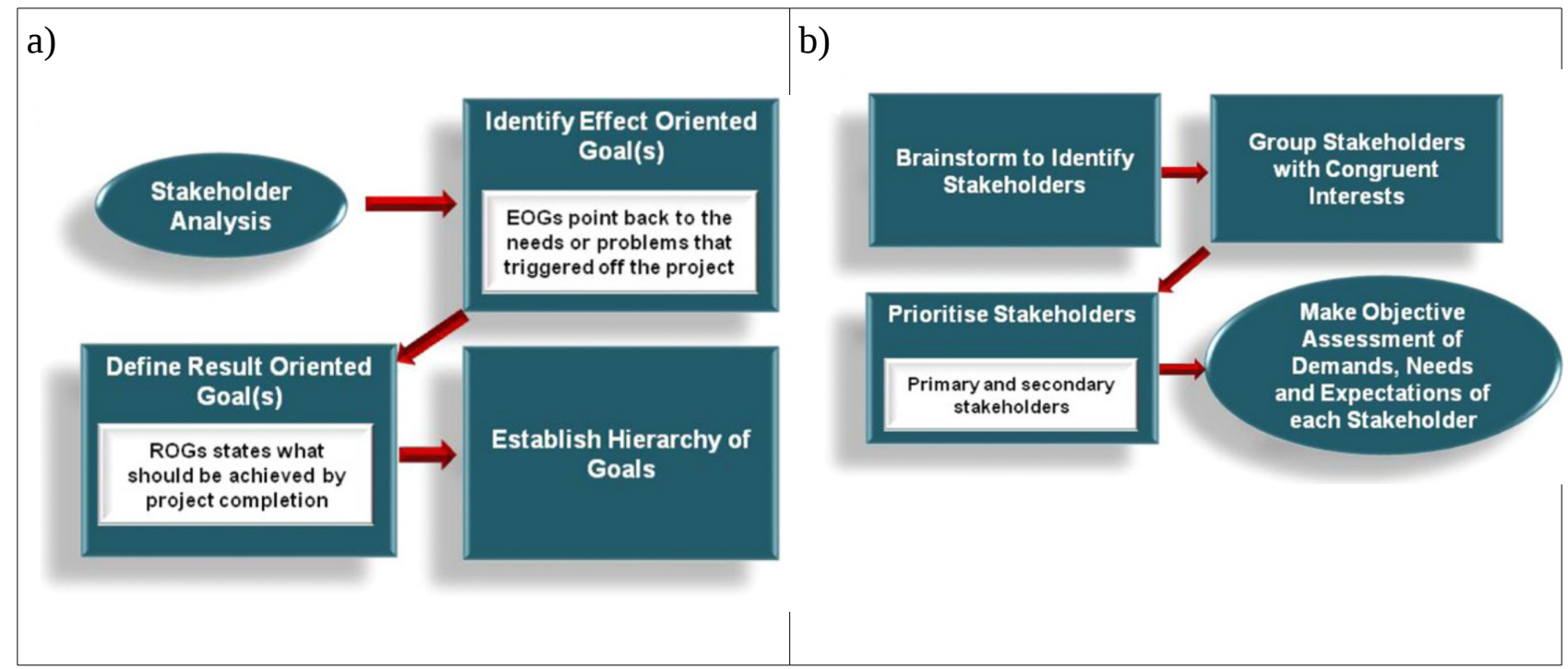

Figure 4: Example of task diagram

The interviews resulted in more than 300 task elements distributed across different areas of project 
management. This data was used primarily to model the task models, as illustrated in Figure 5. It was also used to scope the second phase of interviews. The goal of this exercise was to identify the most difficult and important task areas. We adopted the following structured coding procedure. The first step was to code each task element into one or two categories (each category corresponded to one of the task areas in the original model shown to participants at the beginning of the interview). Most task elements could be easily coded into one category. For the most part, this category was simply the task area the element had been nested under in the task diagram. In other cases, task elements were coded into different categories, either because it seemed the participant had included task elements outside of the task area (e.g. a preceding or subsequent sub-task in an adjoining task area) or because the participant appeared to have misunderstood the semantic meaning of the task

area. In a few cases, task elements were coded into more than one category because the task areas in the original model overlapped. Each task area was then scored in terms of the number of participants who had identified a difficult task element within the area. The coding was carried out by two researchers with experience in project management and validated by a domain expert in project management. The outcome of the coding procedure was an ordered list of the most cognitively challenging task areas.

\section{Knowledge audit and simulation interviews}

The knowledge audit and simulation interviews addressed just those task areas where four or more participants had identified a difficult/important element. These include stakeholder and requirements analysis, human resource management, defining project goals and scope definition. Interviews were carried out with 10 participants in four countries (UK, Finland, Norway and Bulgaria). Potential participants were screened to ensure that they had at least 5 years project management experience. Seven participants were male and three were female. They were aged between 38 and 57 years, with a mean age of 44 years. On average, they had 11 years of project management experience. The interviews were administered by three different interviewers. At the start of each interview, following a brief questionnaire, participants were asked to choose two of four areas identified as important and difficult in Phase 1. These were then addressed first using the knowledge audit protocol. First, probes were used to elicit examples; the examples were then queried for expert cues and strategies and for explanations as to why novices might find the situation difficult. Next, the simulation interview protocol was applied. Interviews lasted between 90 min and $2 \mathrm{~h}$. In some interviews, due to time constraints, one rather than two task areas was addressed.

\section{Knowledge representation}

The output of both phases of interviews was a set of representation frameworks i.e. for each task area addressed by the in-depth interviews we produced a task model, cognitive demand table and a BDI framework. In this section, due to space limitations, we present the output for one of these areas: scope definition. The task model for scope definition is captured in Figure 5. Seven core task components were identified. 


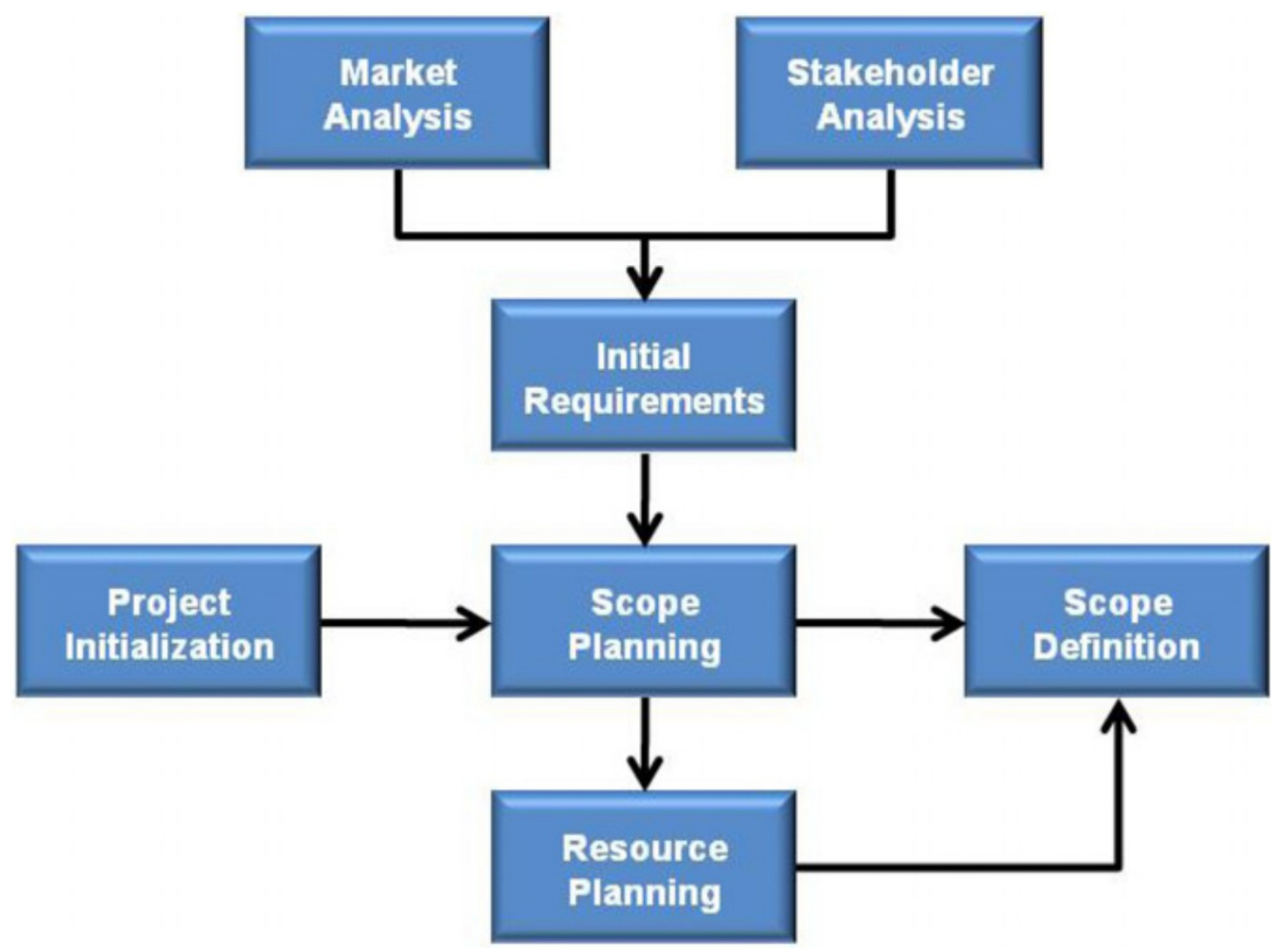

Figure 5: Task model of scope definition.

The data obtained from the knowledge audit and the simulation interviews was coded into the following categories: why difficult, cues and strategies and potential errors. Following the coding, data from each task area were collated together into a cognitive demands table.

Table 1 below presents an extract from the cognitive demands table for scope definition.

\begin{tabular}{|l|l|l|l|}
\hline $\begin{array}{l}\text { Difficult } \\
\text { cognitive } \\
\text { element }\end{array}$ & Why difficult & Potential errors & Cues and strategies \\
\hline $\begin{array}{l}\text { Understanding } \\
\text { the 'real' } \\
\text { requirements }\end{array}$ & $\begin{array}{l}\text { It is common for } \\
\text { stakeholders to be } \\
\text { uncertain about what } \\
\text { they want or they } \\
\text { may have difficulty } \\
\text { articulating what } \\
\text { they want. A novice } \\
\text { might think his or } \\
\text { her job is just to }\end{array}$ & $\begin{array}{l}\text { Taking stakeholder } \\
\text { requirements at face } \\
\text { value and blindly } \\
\text { implement them }\end{array}$ & $\begin{array}{l}\text { An expert engages in a full } \\
\text { dialogue with } \\
\text { stakeholders to try to ensure } \\
\text { that the real } \\
\text { underlying requirements are } \\
\text { being met. }\end{array}$ \\
\cline { 2 - 4 } & $\begin{array}{l}\text { Focusing on delivery } \\
\text { despite signals that }\end{array}$ & $\begin{array}{l}\text { To allow dialogues to take } \\
\text { place, project }\end{array}$ \\
\hline
\end{tabular}




\begin{tabular}{|c|c|c|c|}
\hline & $\begin{array}{l}\text { execute and deliver } \\
\text { rather than consider } \\
\text { what the real } \\
\text { underlying } \\
\text { requirements are. }\end{array}$ & $\begin{array}{l}\text { a particular requirement is } \\
\text { not really } \\
\text { wanted }\end{array}$ & $\begin{array}{l}\text { managers need to ensure } \\
\text { that } \\
\text { relationships with key } \\
\text { stakeholders are } \\
\text { close and immediate. }\end{array}$ \\
\hline $\begin{array}{l}\text { Distinguishing } \\
\text { needs and } \\
\text { implementations }\end{array}$ & $\begin{array}{l}\text { Novices often think } \\
\text { that the specification } \\
\text { has to be thorough, } \\
\text { meaning and that } \\
\text { everything has to be } \\
\text { specified. Also, with } \\
\text { scope and } \\
\text { requirements } \\
\text { definition, it is } \\
\text { often difficult to know } \\
\text { when the } \\
\text { optimum specification } \\
\text { has been obtained. }\end{array}$ & $\begin{array}{l}\text { Including } \\
\text { implementations in the } \\
\text { requirements definition. } \\
\text { Defining how is } \\
\text { requirements definition. } \\
\text { Defining how is } \\
\text { unnecessary and } \\
\text { potentially problematic. } \\
\text { It constrains the project to } \\
\text { a particular } \\
\text { implementation better } \\
\text { might become available, } \\
\text { or the planned } \\
\text { implementation may turn } \\
\text { out to be infeasible }\end{array}$ & $\begin{array}{l}\text { An expert tries to get } \\
\text { stakeholders to specify what } \\
\text { they want but not how to } \\
\text { achieve it. To distinguish } \\
\text { 'what' from } \\
\text { 'how', one strategy is to } \\
\text { look at the project outcomes } \\
\text { from a user perspective i.e. } \\
\text { identify wanted features at a } \\
\text { high level without } \\
\text { specifying technical details. } \\
\text { level without specifying } \\
\text { technical details }\end{array}$ \\
\hline $\begin{array}{l}\text { Ensuring that } \\
\text { requirements can } \\
\text { be } \\
\text { met within } \\
\text { resource } \\
\text { constraints }\end{array}$ & $\begin{array}{l}\text { A novice may be more } \\
\text { eager to please the } \\
\text { external client or the } \\
\text { internal sponsor, } \\
\text { and say 'yes' to } \\
\text { everything rather than } \\
\text { entering into a } \\
\text { negotiation mode. A } \\
\text { novice may lack the } \\
\text { self-confidence to be } \\
\text { assertive and to } \\
\text { negotiate and drive a } \\
\text { bit of a bargain. }\end{array}$ & $\begin{array}{l}\text { Allowing clients to 'over- } \\
\text { egg' the } \\
\text { requirements }\end{array}$ & $\begin{array}{l}\text { Recognise that saying yes } \\
\text { to all requirements can push } \\
\text { the project scope beyond } \\
\text { the available funding and } \\
\text { that a certain amount of } \\
\text { negotiation and/or } \\
\text { renegotiation is usually } \\
\text { necessary. Part of this } \\
\text { (re)negotiation involves } \\
\text { prioritizing } \\
\text { requirements in } \\
\text { consultation with } \\
\text { stakeholders e.g. by getting } \\
\text { clients to } \\
\text { distinguish 'must have' } \\
\text { from 'nice to } \\
\text { have' requirements. }\end{array}$ \\
\hline
\end{tabular}

Table 1: Extract from the cognitive demands table for scope definition.

\section{Implications for design}

To illustrate how the methodology affects the game design, we consider each the difficult cognitive elements presented in previous section. The data suggest a number of possible game features and mechanics for a game scenario targeting competence development in scope definition.

- When eliciting project requirements, the player should interact with a number of non-player characters representing the project stakeholders. Each stakeholder NPC should possess a subset of the overall project requirements. 
- Internally, the game should classify the requirements according to different levels of importance and impact on the project being considered a success.

- Some stakeholders should fail to clearly communicate their 'real' requirements. To support this, the game mechanic of 'position/interest' has been developed. Each stakeholder's position will dictate what he or she is capable of communicating in response to initial probing, whilst each stakeholder's interest will dictate his/her real needs. To elicit the elusive 'real' requirements, players must do more elaborate probing and use deduction.

- Some stakeholder NPCs should associate some of their requirements to specific implementations. Success in the scenario relies on the player ensuring that implementation specifications are not included in the scenario.

- Each requirement will have a perceived level and a real level of importance, and these two do not necessarily match with one another. Some stakeholder NPCs should request requirements that are expensive or difficult to achieve. Players must be prepared to negotiate and resist pressure to include these requirements where they are particularly expensive or difficult to achieve and not, in their view, strictly necessary. This leads to the inclusion of a game mechanic that captures influence. If the player is successful, then the negotiation outcome will be in their favour and the requirement may be reclassified to 'nice to have' instead of 'must have'. However, it is important that before the player exercises their influence, they should suspect that the real level of importance of a requirement is lower than the perceived.

- One or two crucial requirements will be held by a stakeholder NPC that is not listed on the organisational chart. To succeed in the scenario, players must make additional efforts (e.g. talking to other stakeholders) to ensure that all key stakeholders are identified and included in the scope definition process.

- Considering the importance of communication in the process of determining requirements, chatbot technology - namely Artificial Intelligence Markup Language (AIML) - will be used. The alternative of option-based dialogue would be limited, guiding the learner to set of responses and would not instil in the player the importance of how requirements elicitation is carried out.

Ultimately, a player's actions and inactions will be reflected in their scores and/or by events within the game. For example, players who take the initial requirements at face value will risk implementing some unneeded and unwanted requirements whilst missing some important requirements. Similarly, if a player misses an important stakeholder from the requirements analysis, this stakeholder may emerge later in the game scenario, with negative consequences for the project.

\section{Discussion}

At the start of our venture to create a serious game, an initial game design was developed. Our first approach was based on the traditional approach to game design (Figure 1), where design assumptions were derived from the experience of game developers involved in the development, 
and from interaction with five subject matter experts (e.g. lecturers in project management) and several text books in the topic. Similar to other cases (e.g. (Aldrich, 2009)), the output of the elicitation process contributed to deeper understanding of the problem domain, but failed to yield concrete information with clear implications for the game design.

Our initial game design concept focused on project execution. The user would receive a project brief with a given workbreakdown structure (WBS). The driving principle of the serious game would then be the execution of the project, with the various tasks being represented by nodes of a simulation engine. The player would then be challenged with monitoring the progress of the project, and ensuring that tasks were completed on time with the required quality and within the allocated budget. This would imply managing the various stakeholders within the project and dealing with any unexpected issues that emerged, making changes as deemed necessary.

After implementing our methodology for knowledge elicitation and representation, we were surprised to find that the previous tasks were deemed cognitively non-challenging, thus not being appropriate for a serious game. In addition, our approach elicited a great deal of information concerning the types of cues and strategies that people and the kinds of errors that novices typically make. This information is helping the game designers within the project develop the game mechanics that are needed to structure the game design.

Our approach can be applied to any serious game aimed at facilitating competence development within the workplace. Since part of the approach supports identification of difficult and/or important task elements, it is particularly useful for large and complex learning domains where scoping down to a more limited set of learning objectives is required.

As the ACTA method relies on structured interview tools, our approach is well suited to job domains where observational data is difficult to obtain. It is also appropriate for job domains where there is a good deal of variation in the way that tasks are carried out since, unlike some CTA methods (e.g. GOMS), it does not rely on a detailed examination of the procedural elements within the tasks addressed by the game.

Although our approach produced the desired results for project management, it is unclear how well our approach will work in learning domains that are not based around workplace tasks e.g. abstract learning domains such as mathematics.

\section{Conclusion}

Serious games development remains a craft, where success is unpredictable. There is a need for systematic methodologies to elicit, distil and represent expert domain knowledge to increase the probability of success. In this paper, we present a structured approach to game design for serious games based on an extended Applied Cognitive Task Analysis (ACTA) method. We illustrate how the outputs of this approach can inform game flow and mechanics by explaining how they can shape the design of a serious game aimed at reducing time-to-competence in project management.

\section{Acknowledgements}

The research reported in this publication has been undertaken within the TARGET project, which is 
partially funded by the European Community under the Seventh Framework Programme (Grant Agreement 231717). The authors thank all those who participated in the research as participants. The authors would also like to thank Ana Sena from INdT for helping to conduct some of the interviews.

\section{References}

Aldrich, C., 2009. The Complete Guide to Simulations and Serious Games: How the Most Valuable Content Will be Created in the Age Beyond Gutenberg to Google, illustrated. ed, Pfeiffer Essential Resources for Training and HR Professionals. Jossey Bass.

Baber, C., Borras, C., Hone, G., MaCleod, I., MacMaster, R., Salmon, P., Stanton, N., 2005. Cognitive Task Analysis: Current Use and Practice in the UK Armed Forces and Elsewhere.

Baskin, J.D., John, B.E., 1998. Comparison of GOMS analysis methods, in: CHI '98: CHI 98 Conference Summary on Human Factors in Computing Systems. ACM, New York, NY, USA, pp. 261-262. doi:10.1145/286498.286742

Bratman, M.E., 1987. Intentions, Plans, and Practical Reason, The Hume Series. Harvard University Press, Cambridge, MA, USA.

Chipman, S.E., Schraagen, J.M.C., Shalin, V.L., 2000. Introduction to Cognitive Task Analysis, in: Cognitive Task Analysis. Lawrence Erlbaum Associates.

Crandall, B., Klein, G., Hoffman, R.R., 2006. Working Minds: A Practitioner's Guide to Cognitive Task Analysis (Bradford Books), 1st ed. A Bradford Book.

Eck, R.V., 2006. Digital Game-Based Learning: It's Not Just the Digital Natives Who Are Restless. EDUCAUSE Review 41, 16-30.

ESA, 2010. Essential Facts about the Computer and Video Game Industry.

Filho, W.A.D.A., Latham, L., 2006. Key Reasons Why You Should Consider a "Learning by Gaming” Strategy. Gartner.

Jana, R., 2007. Microsoft's Games Get Serious - Businessweek.

Lui, F., Connell, R., Vaughan, J., 2002. An architecture to support autonomous command agents for onesaf testbed simulations, in: In Proceedings of the SimTecT Conference 2002.

Militello, L.G., Hutton, R.J.B., 1998. Applied cognitive task analysis (ACTA): a practitioner's toolkit for understanding cognitive task demands. Ergonomics 1618-1641.

Norling, E., 2003. Capturing the quake player: using a BDI agent to model human behaviour, in: AAMAS '03: Proceedings of the Second International Joint Conference on Autonomous Agents and Multiagent Systems. ACM, New York, NY, USA, pp. 1080-1081. doi:10.1145/860575.860805

Norling, E., 2009. On Evaluating Agents for Serious Games, in: Dignum, F., Bradshaw, J., Silverman, B., van Doesburg, W. (Eds.), Agents for Games and Simulations, Lecture Notes in Computer Science. Springer Berlin Heidelberg, pp. 155-169.

Norling, E., Sonenberg, L., 2004. Creating interactive characters with BDI agents, in: Proceedings of IE2004: Australian Workshop on Interactive Entertainment. Presented at the IE2004, Sydney, Australia.

Paterno, F., Mancini, C., Meniconi, S., 1997. ConcurTaskTrees: a Diagrammatic Notation for Specifying Task Models, in: Proceedings of the IFIP TC13 Interantional Conference on Human-Computer Interaction. Presented at the IFIP, Chapman \& Hall Ltd, London, UK, pp. $362-369$.

Raybourn, E.M., Bos, N., 2005. Design and Evaluation Challenges of Serious Games, in: CHI '05 Extended Abstracts on Human Factors in Computing Systems. Presented at the Conference on Human Factors in Computing Systems, ACM, New York, NY, USA, Portland, OR, USA. doi:http://doi.acm.org/10.1145/1056808.1057094

Vidani, A., Chittaro, L., 2009. Using a Task Modeling Formalism in the Design of Serious Games 
for Emergency Medical Procedures, in: 2009 Conference in Games and Virtual Worlds for Serious Applications. Presented at the VS-Games, IEEE Computer Society, Coventry, UK. doi:http://doi.ieeecomputersociety.org/10.1109/VS-GAMES.2009.24

Zachary, W.W., Ryder, J.M., Hicinthbothom, J.H., 1998. Cognitive Task Analysis And Modeling Of Decision Making In Complex Environments, in: Decision Making Under Stress:

Implications for Training and Simulation. American Psychological Association, Washington, DC, USA. 